\title{
Detecting Particles Flowing through Interdigitated 3D Microelectrodes
}

\author{
Elena Bianchi", Enrica Rollo*, Samuel Kilchenmann, Francesco M. Bellati, Enrico Accastelli and \\ Carlotta Guiducci, Member, IEEE
}

\begin{abstract}
Counting cells in a large microchannel remains challenging and is particularly critical for in vitro assays, such as cell adhesion assays. This paper addresses this issue, by presenting the development of interdigitated three-dimensional electrodes, which are fabricated around passivated pillarshaped silicon microstructures, to detect particles in a flow. The arrays of micropillars occupy the entire channel height and detect the passage of the particle through their gaps by monitoring changes in the electrical resistance. Impedance measurements were employed in order to characterize the electrical equivalent model of the system and to detect the passage of particles in real-time. Three different geometrical micropillar configurations were evaluated and numerical simulations that supported the experimental activity were used to characterize the sensitive volume in the channel. Moreover, the signal-to-noise-ratio related to the passage of a single particle through an array was plotted as a function of the dimension and number of micropillars.
\end{abstract}

\section{INTRODUCTION}

Fluidic, chemical and sensing functions can be integrated on lab-on-a-chip devices with the purpose of miniaturizing and automatizing laboratory practices and analytical processes. Pushing miniaturization and performance of sensors to the extreme makes it possible to reach single cell level. Electrical-based sensing techniques have been widely employed in this field due to their amenability to be implemented as parallel measurement systems and for their advantages related to label-free and non-destructive detection with respect to fluorescence techniques [1].

For cell counting applications, miniaturized devices based on the Coulter counter techniques have been recently introduced to address the need for parallelism and portability. For instance, microfluidic Resistive Pulse Sensors (RPS) lead to easy miniaturization and improved Signal-to-Noise-Ratio (SNR) [2]. Nevertheless, their limiting factor is the low

Research supported by the Swiss Federal Institute of Technology Lausanne $(\mathrm{CH})$ and by the swissUP Foundation.

"Authors contributed equally to the paper.

E. Bianchi is with the Swiss Federal Institute of Technology, Lausanne (EPFL), Switzerland and with the Politecnico di Milano, LaBS, Laboratory of Biological Structure Mechanics, Milano (I) (elena.bianchi@epfl.ch).

E. Rollo is with the Institute of Bioengineering, Swiss Federal Institute of Technology, Lausanne (EPFL), Switzerland. ; e-mail: enrica.rollo@epfl.ch).

S. Kilchenmann is with the Institute of Bioengineering, Swiss Federal Institute of Technology, Lausanne (EPFL), Switzerland (e-mail: samuel.kilchenmann@epfl.ch).

F. M. Bellati is with the Politecnico di Milano, LaBS, Laboratory of Biological Structure Mechanics, Milano (I) (francesco.bellati@epfl.ch).

C. Guiducci is with the Institute of Bioengineering, Swiss Federal Institute of Technology, Lausanne, Switzerland (EPFL) corresponding author. (phone: +41216937813 ; e-mail: carlotta.guiducci@epfl.ch). throughput coming from the use of a single micro- or submicro channel resulting in a low flow-rate [3]. Recently, multi-aperture Coulter counters have been introduced to enable parallel operations [4].

In the field of elelctrical-based cell counters, several recent works witness a wide interest in the use of the Impedance Spectroscopy (IS) technique for the development of miniaturized systems [5]. The sensing principle is based on the impedance change induced by the presence of a cell between two electrodes. This change is due to the dielectric properties of the cells, determined by the presence of the highly insulating bilayer membrane. Impedance-based systems have been used to monitor the cell growth and viability [6], and to identify cells affected by diseases such as cancer [7] or malaria [8]. These studies are performed either on cells in a flow [9] or on cells adhered on co-planar electrodes [10] [11]. Previous works performing IS on suspended particles are based on channels or apertures characterized by a flow section comparable to the dimension of the particles under test ([12], [13], [14], [15], [16]). Gawad et al. demonstrated the possibility to differentiate and count cells in a channel by co-planar microfabricated Pt electrodes [12]. Facing electrode configurations in a channel were proposed to improve the impedance signal caused by the passage of a particle [13] [14] [17]. Indeed, since the generated field of vertical facing-electrodes is uniformly distributed, the impedance change is not affected by the position of the cell in the z-axis.

In this paper we address the detection of cells flowing through parallel-plate flow-chambers with large width-height ratio. These microfluidic chambers are employed in flowdependent cell adhesion assays, where the ability of cells to adhere to a surface is evaluated in controlled shear-stress conditions. For instance, they have been employed to observe under flow conditions the adhesion and migration through the endothelium of leucocytes [18] and of melanoma cells in presence of neutrophils [19].

A possible strategy to perform adhesion tests is to count cells before and after the passage through the adhesion chamber in order to evaluate the number of adhered cells. Nevertheless, for this application the drastic flow perturbations caused by cells passing through a small aperture should be avoided. To overcome this problem, we developed interdigitated pillar-shaped microelectrodes to be distributed along the flow section of the parallel-plate flowchamber, implementing two sensors, one close to the inlet and the other close to the outlet.

In our approach, each single impedance sensor is 
constituted by two or more pillar-shaped microelectrodes arranged along a line normal to the flow direction (Fig. 1a). The electrodes are connected in an interdigitated fashion to only two pads. Compared to a solution employing series of electrode couples along the channel width, our system avoids capacitive coupling between the wires of the different adjacent sensors and drastically reduces the number of pads. Overall, the adopted solution is suitable for the implementation of on chip devices featuring parallel assays.

In this paper we present experimental results on two- and three-pillar sensors of different dimensions, tested on the detection of polystyrene beads in flow. Moreover, we support our results with numerical simulations to optimize the design for single-particle counting and to investigate the theoretical limits of the presented devices.

\section{MATERIALS AND METHODS}

\section{A. Experimental characterization by impedance measurements}

\section{1) Chip assembly}

Vertical platinum electrodes were obtained by metal deposition on a passivated silicon substrate on which pillarshaped structures were previously fabricated. The electrodes were connected to external pads by wires that were passivated with silicon dioxide. For all devices, only two pads were used to contact the pillars which were connected in an alternate configuration, implying that the equivalent electrical elements of the volumes in between the pillars are connected in parallel (Fig. 1a).

A PDMS (polydimethylsiloxane, Sylgard 184 DowCorning) cover was bonded to the silicon substrate to form a $50 \mu \mathrm{m}$ high microfluidic channel where beads are carried in a laminar flow of buffer solution. Three different micropillar configurations have been designed for the impedance spectroscopy experiments.
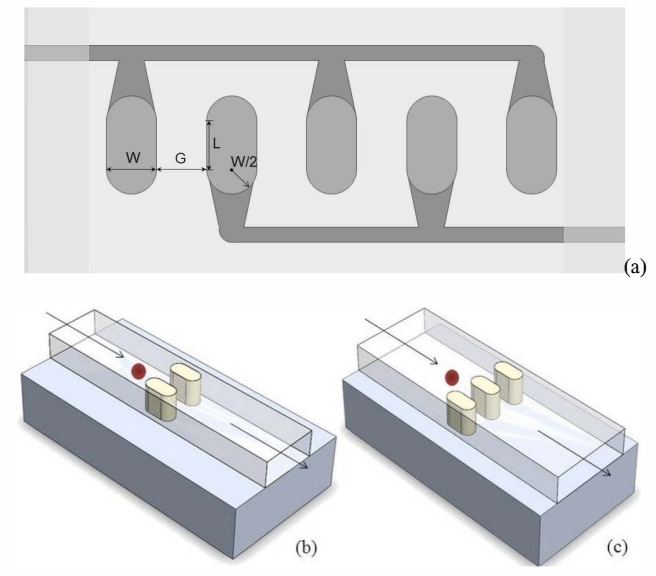

Figure 1. a) Top view of the interdigitated sensor constituted by the array of pillar-shaped microelectrodes, $W$ is the width of the micropillar, $G$ is the distance between the micropillars and $\mathrm{L}$ is the length of the electrode surfaces parallel to the channel sidewalls. b-c) 3D sketches of the chip assembly with either two (b) or three (c) micropillars in the channel. All micropillars and channels are $50 \mu \mathrm{m}$ high. All pillars have a identical length dimension $\mathrm{L}$ in the direction of the flow.

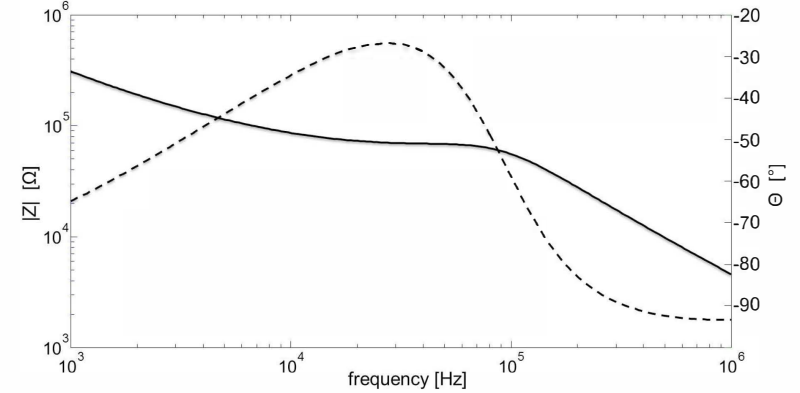

Figure 2. Bode plot of the two-pillar $30 \mu \mathrm{m}$-configuration. The plateau of the impedance (solid line) appearing in the $10^{4} \mathrm{~Hz}-10^{5} \mathrm{~Hz}$ range corresponds to the resistive parameter of the solution in between the electrodes. Dashed line represents the phase.

The electrodes were designed to occupy $50 \%$ of the channel flow section, thus the width of the micropillars (W) is equal to the gap in between them $(\mathrm{G})$ ranging from $30 \mu \mathrm{m}$ to $50 \mu \mathrm{m}$ (Fig. 1a). Moreover, the length dimension $\mathrm{L}$ of the micropillars is constant for the three geometries and equal to $30 \mu \mathrm{m}$. The three geometrical configurations will be indicated in the following as: $30 \mu \mathrm{m}-40 \mu \mathrm{m}$ - and $50 \mu \mathrm{m}$-configuration. As shown in Fig. 1, front and back faces of the micropillars are rounded to ensure a better hydrodynamic behavior by reducing the presence of stagnation zones where beads could be trapped.

\section{2) System instrumentation}

The impedance measurements were performed using an Agilent 4294A impedance analyzer remotely controlled by a LabVIEW interface. A PCB holds the chips and provides connection to the instrument. A syringe pump (Harvard, Milliliter syringe pump module OEM) was used to generate flow-rates in the range of $0.5 \mu \mathrm{L} / \mathrm{min}-1 \mu \mathrm{L} / \mathrm{min}$ to move the beads along the channel. The setup was mounted on a Leica DM2500 microscope and a synchronized video of the channel was recorded with a Leica DFC295 camera at $23 \mathrm{frame} / \mathrm{s}$, in order to simultaneously observe the impedance signal and the bead position.

\section{3) Impedance measurements}

Impedance characterization of the chips has been performed both in air and in 0.1X PBS (Phosphate Buffered Saline, Sigma Aldrich). The sinusoidal amplitude was set at $50 \mathrm{mV}$ and the bandwidth of the tool to 3 . The silicon chip was connected to ground to eliminate capacitive couplings between the wires and the silicon substrate. Bode plots of our systems were obtained by sweeping the frequency over 801 measurement points logarithmically distributed between $10^{3} \mathrm{~Hz}$ and $10^{6} \mathrm{~Hz}$. For other experiments, consecutive single-frequency measurements at $40 \mathrm{kHz}$ and $80 \mathrm{kHz}$ have been performed to detect the passage of $10 \mu \mathrm{m}$ polystyrene beads ( $200-500$ beads $/ \mu$ l, Sigma Aldrich) in real-time through the sensing electrodes. The impedance was recorded at time intervals of $25 \mathrm{~ms}$.

\section{B. Numerical simulations of impedance characteristics}

Comsol Multyphysics was used to simulate the impedance response of the system to an $\mathrm{AC}$ voltage input [20]. A tetrahedral grid, consisting of $170^{\prime} 000$ to $300^{\prime} 000$ elements, according to the considered geometrical configuration, was applied on the volume. Smaller structures, 
such as electrochemical double layer or passivation layers, were represented as interfaces.

In this model, we consider the equation of continuity:

$$
\nabla \cdot J=0
$$

where $J\left[\mathrm{~A} / \mathrm{m}^{2}\right]$ is the current density, and the constitutive Ohm law:

$$
\boldsymbol{J}=\left(\sigma+j \omega \varepsilon_{0} \varepsilon_{r}\right) \mathbf{E}
$$

with $\sigma[\mathrm{S} / \mathrm{m}]$ the conductivity, $\omega[\mathrm{rad} / \mathrm{s}]$ the angular frequency, $\varepsilon_{0}[\mathrm{~F} / \mathrm{m}]$ the permittivity of vacuum, $\varepsilon_{\mathrm{r}}$ the relative permittivity, and $E[\mathrm{~V} / \mathrm{m}]$ the electric field. The electrical properties of the materials that were applied in the simulations are reported in Table 1.

Table.1 Electrical parameters applied in the numerical simulation.

\begin{tabular}{|c|c|c|}
\hline Material & $\boldsymbol{\sigma}[\mathbf{S} / \mathbf{m}]$ & $\boldsymbol{\varepsilon}_{r}$ \\
\hline Silicon & 100 & 11.6 \\
\hline Silicon dioxide & $10^{-8}$ & 4.5 \\
\hline Platinum & $9.910^{6}$ & - \\
\hline PBS 0.1X & 0.15 & 78 \\
\hline PDMS & - & 3.8 \\
\hline Polystyrene & $10^{-16}$ & 2.6 \\
\hline
\end{tabular}

Interfaces have been modeled as lumped parameter elements.

A sinusoidal signal with an amplitude of $50 \mathrm{mV}$ was applied to the external terminals. A set of 50 frequency points ranging from $1 \mathrm{~Hz}$ to $10^{12} \mathrm{~Hz}$ was considered for the parametric study. In agreement with the realized devices, numerical simulations of sensors constituted of several pillars are considered as two main interdigitated electrodes.

Impedance module $(|\mathrm{Z}|)$ and phase $(\theta)$ were calculated for each micropillar configuration in air and in wet conditions (PBS 0.1X buffer solution) and in the presence of a bead between the electrodes. Moreover, the impedance changes caused by a bead passing along the centerline in between two micropillars was simulated at nine different positions. The computational grid was identical for each bead position in order to avoid the effect of grid changes on the results [21].
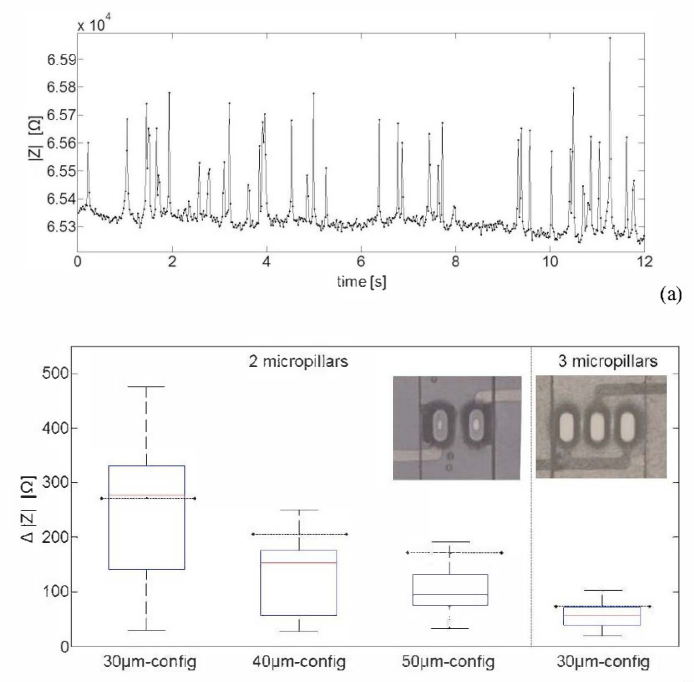

(b)

Figure 3. a) Raw data of impedance measurements at $40 \mathrm{kHz}$ for the $30 \mu \mathrm{m}$ configuration observed in real-time under flow conditions. b) The heights of the measured peaks are reported for sensors with two pillars (left section) and with three pillars (right section). The geometrical configurations are indicated on the $\mathrm{x}$ axis. The plot summarizes the results corresponding to 389 events. Dotted lines report the simulated maximum of the peaks.

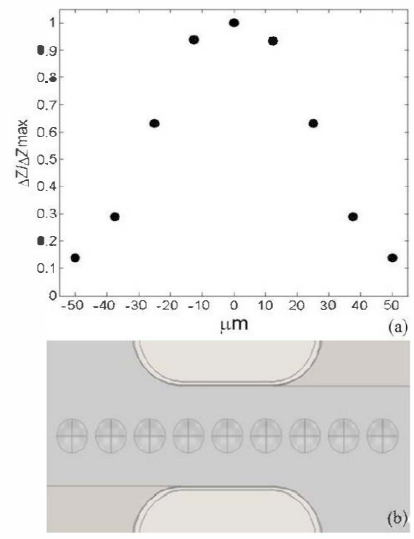

Figure 4. Numerical simulations of nine different stages of the passage of a bead through two micropillars in the $30 \mu \mathrm{m}$-configuration.

\section{RESULTS AND DISCUSSION}

\section{A. Impedance spectra}

The Bode plot of the $30 \mu \mathrm{m}$-configuration is shown in Fig. 2. At low frequencies, the spectrum is dominated by the electrode/solution interface capacitance and at medium frequencies $\left(10^{3} \mathrm{~Hz}\right.$ to $\left.10^{5} \mathrm{~Hz}\right)$ by a resistive plateau, which is affected by the electrolyte solution resistance between the micropillars and by passing beads. The interface capacitance extracted from the Bode plot of different chips and electrode configurations ranges from $11 \mu \mathrm{F} / \mathrm{cm}^{2}$ to $15 \mu \mathrm{F} / \mathrm{cm}^{2}$, in good agreement with the values reported in literature for other microfabricated Pt electrodes [22] [23]. The parasitic capacitance between the metal electrodes and the wires has been estimated by simulation in range of $80 \mathrm{fF}-100 \mathrm{fF}$, which implies that it can only be observed at frequencies higher than $10^{8} \mathrm{~Hz}$.

\section{B. Real-time detection of particles in a flow}

Our system has been characterized by employing polystyrene beads that show similar insulating behavior as cells in the frequency range of interest. Consecutive singlefrequency impedance measurements have been performed for all the three geometrical configurations.

The real-time $|Z|$ measurements of the $30 \mu \mathrm{m}$ configuration show several peaks exceeding the baseline significantly (Fig. 3a). Microscope video recordings of the micropillars allow to clearly correlate each peak with the passage of a bead. The value of the impedance peaks measured is smaller than its actual maximum; this is due to the limited sampling rate of the instrument which allows to record only one or two measurement points per peak. The noise is determined by the instrument accuracy, and corresponds to $0.01 \%$ of the measured value.

The distribution of all measured impedance changes for the three configurations of two-pillar devices and for the $30 \mu \mathrm{m}$-configuration of the three-pillar devices. The signal due to the passage of a bead is higher in the case of the $30 \mu \mathrm{m}$-configuration with respect to the other two configurations with larger gaps and pillars. 


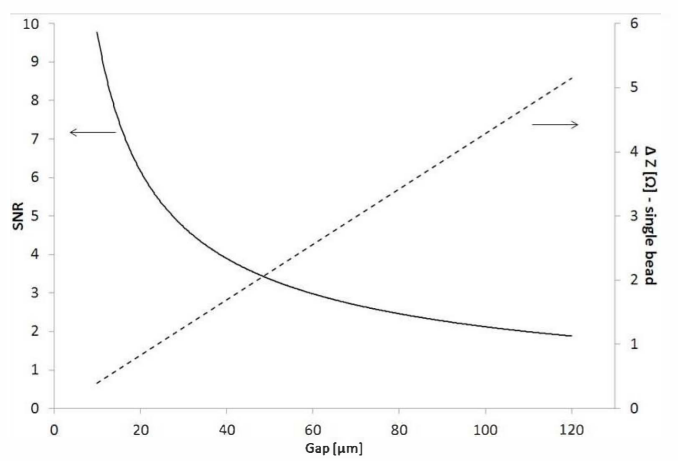

Figure 5. The right axis represents the impedance change upon the passage of a bead through $1 \mathrm{~mm}$ wide flow section as a function of the dimension of the pillars. SNR on the left axis shows the decrease of the signal to noise ratio with the increasing dimension of the pillars/gaps.

Moreover, the three-pillar device detects a four times smaller impedance change. A similar behavior was found in simulations. In particular, the simulated values of the peak maximum for the different devices describe a similar trend as the experimental results (Fig 3b).

In order to estimate the impedance change during the passage of a bead in between the micropillars, simulations have been performed for the two-pillar $30 \mu \mathrm{m}$-configuration. The results show that the pillars provide a $100 \mu \mathrm{m}$ long sensing volume along the flow direction where the signal of the bead is at least $15 \%$ of its maximum value (Fig. 4 ).

3D simulations were also employed to characterize the impedance change due to one single bead as a function of the pillar/gap dimensions in a $1 \mathrm{~mm}$-wide array of identical pillars. Considering a fixed channel width, increasing equally the gap dimension and the width of the pillars will lead to a reduction of the number of pillars fitting in the array. A lower number of gaps in the flow section is more favorable for the detection of a single bead passing through one of the gaps. This suggests that larger pillars and gaps lead to a higher impedance signal due to the bead (Fig. 5 right axis). Nevertheless, larger gaps and larger pillars correspond to a higher value of the resistive plateau resulting in a significantly higher absolute instrumental error. By combination of the results of the simulations and the evaluation of the noise, we derived that the increase of the pillar dimension and gaps lead to a decrease of the SNR (Fig. 5 left axis).

It is possible to derive that in order to have a SNR greater than 3 , the length of a $30 \mu \mathrm{m}$-configuration array should not exceed $1.6 \mathrm{~mm}$ with the present measurement setup.

\section{CONCLUSIONS}

The present work shows the suitability of 3D microelectrode arrays to detect the passage of single particles flowing in flow-through chambers, via both experimental data and numerical simulations on polystyrene beads. On the other hand, the detection of multiple events is still to be investigated.

Our actual setup is limited by the accuracy of the impedance analyzer corresponding to a percentage relative error of 0.01 on the measured impedance and by the low sampling rate. Therefore, the performance of the system could be further improved by ad hoc circuitry.

\section{REFERENCES}

[1]H.-T. Chen and Y.-N. Wang, "Optical microflow cytometer for particle counting, sizing and fluorescence detection," Microfluidics and Nanofluidics, vol. 6, pp. 529-537, 2009.

[2]X. Wu, et al., "Microfluidic differential resistive pulse sensors," ELECTROPHORESIS, vol. 29, pp. 2754-2759, 2008.

[3]H. Zhang, et al., "Methods for counting particles in microfluidic applications," Microfluidics and Nanofluidics, vol. 7, pp. 739-749, 2009.

[4] V. J. Ashish, et al., "Detection and counting of micro-scale particles and pollen using a multi-aperture Coulter counter," Measurement Science and Technology, vol. 17, p. 1706, 2006.

[5]S. Gawad, et al., "Impedance Spectroscopy and Optical Analysis of Single Biological Cells and Organisms in Microsystems," in Microengineering in Biotechnology. vol. 583, M. P. Hughes and K. F. Hoettges, Eds., ed: Humana Press, 2010, pp. 149-182.

[6]A. R. A. Rahman, et al., "A micro-electrode array biosensor for impedance spectroscopy of human umbilical vein endothelial cells," Sensors and Actuators B: Chemical, vol. 118, pp. 115-120, 2006.

[7]X. B. Wang, et al., "Dielectrophoretic manipulation of cells with spiral electrodes," Biophysical Journal, vol. 72, pp. 1887-1899, 1997.

[8]P. Gascoyne, et al., "Microsample preparation by dielectrophoresis: isolation of malaria," Lab on a Chip, vol. 2, pp. 70-75, 2002.

[9]K. Asami, et al., "Dielectric properties of mouse lymphocytes and erythrocytes," Biochimica et Biophysica Acta (BBA) - Molecular Cell Research, vol. 1010, pp. 49-55, 1989.

[10] R. Ehret, et al., "Monitoring of cellular behaviour by impedance measurements on interdigitated electrode structures," Biosensors and Bioelectronics, vol. 12, pp. 29-41, 1997.

[11] Z. Zhiwei, et al., "A polymer cell chip with in situ monitoring capability of cell viability for cadmium cytotoxicity test of human rhabdomyosarcoma cells," in Solid-State Sensors, Actuators and Microsystems Conference, 2009. TRANSDUCERS 2009. International, 2009, pp. 61-64.

[12] S. Gawad, et al., "Micromachined impedance spectroscopy flow cytometer for cell analysis and particle sizing," Lab on a Chip, vol. 1, p. 76, 2001.

[13] S. Gawad, et al., "Fabrication of a microfluidic cell analyzer in a microchannel using impedance spectroscopy," in Microtechnologies in Medicine and Biology, lst Annual International, Conference On. 2000, 2000, pp. 297-301.

[14] H. Morgan, et al., "Single cell dielectric spectroscopy," Journal of Physics D: Applied Physics, vol. 40, pp. 61-70, 2007.

[15] D. Holmes, et al., "Leukocyte analysis and differentiation using high speed microfluidic single cell impedance cytometry," Lab on a Chip, vol. 9, p. $2881,2009$.

[16] R. Rodriguez-Trujillo, et al., "High-speed particle detection in a micro-Coulter counter with two-dimensional adjustable aperture," Biosensors and Bioelectronics, vol. 24, pp. 290-296, 2008.

[17] K. Cheung, et al., "Impedance spectroscopy flow cytometry: On-chip label-free cell differentiation," Cytometry Part A, vol. 65A, pp. 124-132, 2005.

[18] R. Alon and M. L. Dustin, "Force as a Facilitator of Integrin Conformational Changes during Leukocyte Arrest on Blood Vessels and Antigen-Presenting Cells," Immunity, vol. 26, pp. 17-27, 2007.

[19] S. Liang, et al., "Shear stress and shear rate differentially affect the multi-step process of leukocyte-facilitated melanoma adhesion," Experimental Cell Research, vol. 310, pp. 282-292, 2005.

[20] E. B. Bianchi, Federica; Dubini, Gabriele; Guiducci, Carlotta, "Model of an Interdigitated Microsensor to Detect and Quantify Cells Flowing in a Test Chamber," in 6th annual COMSOL Conference, Paris (F), 2010.

[21] S. Gawad, et al., "Dielectric spectroscopy in a micromachined flow cytometer: theoretical and practical considerations," Lab on a Chip, vol. 4, pp. 241-251, 2004

[22] A. Norlin, et al., "Investigation of interfacial capacitance of Pt, Ti and TiN coated electrodes by electrochemical impedance spectroscopy," Biomolecular Engineering, vol. 19, pp. 67-71, 2002.

[23] S. I. Franks W, Schmutz P, Hierlemann A., "Impedance characterization and modeling of electrodes for biomedical applications.," IEEE Trans Biomed Eng., vol. 52, p. 7, 2005. 